Original Article

\title{
Protocol for a Systematic Review of Rationing of Nursing Care
}

\section{Lata Mandal ${ }^{1}$, A. Seethalakshmi ${ }^{2}$, Anitha Rajendra Babu ${ }^{3}$}

${ }^{1}$ PhD Research Scholar, ${ }^{2}$ PhD Nursing \& Reader, Faculty of Nursing, Sri Ramachandra Medical Centre \& Research Institute, Chennai $-600116,{ }^{3}$ PhD Nursing \& Professor \& Principal, Rajalakshmi College of Nursing, Chennai.

Corresponding author : Lata Mandal, PhD Full time Research Scholar, Faculty of Nursing, Sri Ramachandra M edical Centre \& Research Institute, Chennai -600 116, India. Mobile: +919831483508 E-mail : latakrishnendu@yahoo.com.

Received

: 05.02.2018

Review Completed : 10.03.2018

Accepted

:20.03.2018

Keywords : Rationing, nursing care, missed care, unmet care, patient outcomes

\begin{tabular}{|c|}
\hline Access this article online \\
\hline Quick Response Code \\
\hline
\end{tabular}

\begin{abstract}
Purpose : To identify and synthesize the findings of studies related to "rationing of nursing care". Methods : Preferred Reporting Items for Systematic Reviews and Meta analysis (PRISMA) guidelines will be followed in this review and literature searches will be conducted in M EDLINE, CINAHL, Psyc Info, Web of Science and EMBASE databases. Only quantitative studies meeting the predetermined inclusion criteria, quality standards and credibility will be synthesised using narrative synthesis.
\end{abstract}

Results : A descriptive synthesis of the findings of the selected studies will be carried out which will be presented in narrative summary with statistical find ings incorporated.

Conclusion : This review will provide a synthesis of up to date evidence regarding the rationing of care by nurses. It will also support the hypothesis that rationing of care has effects on patients and nurses and explore relationship of rationing of care with patients and nursing outcome.

\section{Introduction}

Since the last two decades nurse researchers across the world have been studying the concept of "Rationing of care." The findings of these studies suggest that nursing care rationing is widespread ${ }^{1-3}$. However, the levels of rationing reported across studies showed inconsistency, ${ }^{4,5}$. As a concept, "rationing of care" is considered to be relatively new as most of the researches done in this area were in the last decade although sporadic researches on nurses missing and leaving care undone were being studied earlier. A secondary analysis of nursing survey data collected in 1998 have recorded that a very high percentage of nurses left necessary nursing works undone when reported in hospitals across five countries. ${ }^{6}$ There have been studies in the past that have tentatively thrown light on specific aspects of nursing care being omitted or missed. ${ }^{6-9}$ However, it was Dr Beatrice Kalisch of USA who was the first to study this concept of care rationing within the perspective of "Nursing care". 2 Since then many researchers have studied the concept in the context of
"Patient safety" \& "quality of care" and have called it as "M issed care" in nursing. ${ }^{3,10,11-14}$ Independent to their works but somewhat during the same time nurse researchers from European countries were studying the same phenomena and called it "Implicit Rationing of nursing care". They studied the phenomena within the framework of nursing workload and decision making. ${ }^{1,5,15,16}$ Researchers from the Scandinavian countries have also studied the phenomena of nursing care rationing but from the perspective of ethics and justice of care $\&$ called it as "prioritization of care" ${ }^{17-21}$.W Whatso ever may it be named but the commonality of all these researches lie in the ground that rationing have been linked with two very important aspect of health care, namely "quality of nursing care" and "patient's safety and satisfaction".

The existing systematic review on this to pic by Papastavrou is more than five years old. ${ }^{22}$ This review will build upon that research systematically consolidating the level of rationing, aspects of rationing, factors influencing rationing and its impact on nurses and patients across countries. Thus the 
evidence synthesis of this review will help to understand "rationing of nursing care" \& identify evidence gap. This review will further help to inform policies and programmes pertaining to nursing and patient care.

\section{Conceptual definition of rationing of nursing care}

Dr Schubert has studied rationing within the conceptual framework of nurses' decision making and has defined it as "the withholding of or failure to carry out necessary nursing measures for patients due to lack of nursing resources such as staffing, skill mix, or time". ${ }^{1}$ This framework places environmental components like availability of time and resources as pivotal factors influencing nurses doing rationing of care. ${ }^{1}$

Dr Kalisch studied the phenomena within the conceptual framework of patient safety and errors of omission. She called it "missed care" in nursing and indicated it being influenced by both external as well internal factors like individual nurses' beliefs, habits and decision making.

"Prioritization" and "Priority setting" are some other terms used by researchers who focused on the nurses' organization of their work in order to maximize patient outcomes.

"Unmet nursing care needs "and "unfinished care" are other terms used by researchers for "Rationing of nursing care" and have served as Quality indicators for nursing care. ${ }^{4,23}$ for ease of reference this review will be using the term "rationing of nursing care" throughout the text. The others terms will be used while discussing the corresponding literature.

The review will attempt to answer questions regarding:
A) Levels of "Rationing of nursing care"
B) Aspects of nursing tasks that are being rationed
C) Reasons that influence rationing of nursing care
D) Potential effect of rationing on Nurses and patients

\section{Methods}

Preferred Reporting Itemsfor Systematic reviewsand M eta analysis protocol-P 2015 (PRISM A) guidelines were used to prepare the protocol of the review. ${ }^{24}$ This protocol is already registered with PROSPERO International Prospective register for systematic reviews (CRD 42018088679).

Search strategy: Search of literature for this systematic review will follow three steps without any restriction on the date of publication.

1. Initially only M EDLINE and CINAHL plus will be searched with the keywords. The titles and the abstracts from the above search will be analysed looking for additional keywords.

2. In the second step a detailed search will be conducted with all identified keywords in the following databases namely, Psyc Info, Web of Science, EM BASE in addition to CINAHL plus and M EDLINE.

3. In the last step searching of the references of all selected studies will be carried out to lookfor more researches.

\section{Inclusion criteria}

Studies should meet the following criteria to become eligible to be included in the review.

a) Researches published only in peer reviewed journals

b) Available in electronic databases

c) Study design: only quantitative studies will be included in the review

d) Intervention: Studies that explicitly deal with rationing of nursing care as the main variable will be included in the review.

e) Population: Studies must have samples including nurses of any level and experience across all genders, age, race, country or religion.

f) Settings: Conducted in an acute or chronic care clinical setting

g) Outcomes: Studies will be included if they describe either or all of the following variables

(I) Level of rationing,

(ii) Aspects of rationing,

(iii) Factorsinfluencing rationing,

(iv) Rationing and its effect on patients or/and nurses.

h) Language: English only articles

I) Studies that have used the terms "missed care", 
"Unfinished nursing care", "Unmet nursing care needs" will be included even if they do not explicit the definition of "rationing of nursing care."

\section{Exclusion criteria}

A) Studies that have used mixed samples of nurses with other health care providers will be excluded.

B) Conference abstracts, books \& grey literature will be excluded

\section{Screening}

The search titles will be uploaded in Mendeley software and scrutinised to look for duplicates. In the first round titles will be screened as per relevance to the review topic. After this the abstracts of the relevant titles will be read by both the reviewers to screen as per the predetermined inclusion criteria. Following this full text screening will be done as per eligibility criteria. The screening process at both the levels (abstract \& full text) will be done independently by the reviewers and any disagreement will be resolved by discussion and consultation with a third reviewer.

Data extraction: The data extraction tool recommended by the JBI (Joana Brigg's Institute) manual will be used for extracting data from the screened studies. This will include details about the journal of publication, review authors, population characteristics, settings where the study was conducted, design of the study, sample, sampling methods, measurement instruments, validity $\&$ reliability of the instruments, method of data analysis and results. ${ }^{25}$

\section{Criteria for Quality appraisal of the screened studies}

This review will aim at synthesizing data from studies that are of high quality. Therefore each study selected for review will be assessed for its methodological standard. The MAStARI critical appraisal tools recommended by the

\section{References}

1. SchubertM, AusserhoferD, Desmedt M, Schwendimann R, Lesaffre E, $L i B$, et al. Levels and correlates of implicit rationing of nursing care in Swiss acute care hospitals-a cross sectional study. International Journal of Nurs Stud. 2013 Feb; 50(2):230-9. https://doi.org/ 10.1016/j.ijnurstu.2012.09.016

2. Beatrice J Kalisch. M issed Nursing care:a qualitative study. J Nurs Care Qual.2006;21(4):306-13. DOI:10.1097/00001786-200610000-00006

3. Kalisch BJ, Landstrom GL, Hinshaw AS. Missed nursing care:a concept
Joanna Briggs Institute Manual will be used for this purpose. Two independent reviewers will do the appraisal separately and take decision regarding the inclusion of the study for review. Any disagreement on this will be resolved through discussion and consultation with a third reviewer.

\section{Results}

The findings of the studies will be grouped under each review question and presented in the same order as the review questions are mentioned in the protocol. A descriptive synthesis will be carried out and will be presented as a narrative summary in a tabular form under each research question. Both narrative description as well as statistical findings of studies will be used in the summaries. This review will not be pooling statistical findings of various studies for M eta analysis.

\section{Discussions}

Rationing of nursing care is in direct contradiction to Holistic nursing thus creating a dent in "quality nursing care". Therefore an up to date evidence synthesis on "rationing of care" will help the nursing community to identify the commonality \& differences in the levels, aspects, reasons and effects of rationing across studies done in various parts of the world and at different times. Moreover this review will be able to discuss the methodological strength and limitations of the various studies in researching "Rationing in care".

\section{Conclusion}

This review will help the nursing community in general and individual nurses in particular to understand the extent and effect of "rationing of nursing care". It will also help in policy decisions for practice and will identify gaps in the existing literature and suggest areas for further research.

analysis. J Adv Nurs. 2009;65(7):1509-17. https://doi.org/10.1111/ j.1365-2648.2009.05027.x

4. Lucero RJ, Lake ET, Aiken LH. Nursing care quality and adverse events in US hospitals. J Clin Nurs.2010;(19): 2185-2195. https://doi.org/ 10.1111/j.1365-2702.2010.03250.x

5. Schubert M,Glass Tr,Clarke SP,et al. Rationing of Nursing care and its relationship to patient outcomes:the swiss extension of the International hospital outcomes study.Int J Qual health Care 
2008;20(4):227-237

6. Aiken LH, Clarke SP, Sloane DM, Sochalski JA, Busse R, Clarke $H$, Giovannetti P,Hunt J, Rafferty AM, Shamian, J.Nurses' reports on hospital care in five countries. Health Affairs.2001; 20:43-53. https:// doi.org/10.1377/hlthaff.20.3.43

7. Callen BL, Mahoney JE, CB G, TJ W, Enloe M. Frequency of hallway ambulation by hospitalized older adults on units of an academic hospital. Geriatr Nurse. 2004;25(4):212-7. https://doi.org/ 10.1016/j.gerinurse.2004.06.016

8. Rasmussen HH et al. Prevalence of patients at nutritional risk in Danish hospitals. Clin Nutr. 2004;23(5):1009-15. https://doi.org/10.1016 /j.clnu.2004.01.001

9. Forster $\mathrm{A} J$ et al. The Incidence and severity of adverse events affecting patients after discharge from the hospital. Ann Intern Med. 2003:138:161-7. DOI: 10.7326/0003-4819-138-3-200302040-00007

10. Kalisch BJ,Landstrom G,Williams RA.Missed nursing care:errors of omission.Nursing Outlook.2009;57(1):3-9 https://doi.org/10.1016/ j.outlook.2008.05.007

11. Kalisch BJ, Tschannen D, Lee KH. Missed nursing care, staffing, and patient falls. J Nurs Care Qual. 2012 M ar; 27(1):6-12. https://doi.org/ 10.1097/NCQ.0b013e318225aa23

12. Kalisch BJ, Gosselin K, Choi SH. A comparison of patient care units with high versus low levels of missed nursing care. Health Care M anage Rev. 2012 Dec; 37(4):320-8. https://doi.org/10.1097/HM R.0b013e31824 9727 e

13. Winsett RP, Rottet K, Schmitt A, Wathen E, Wilson D, M issed Nursing Care Collaborative Group. Medical surgical nurses describe missed nursing care tasks-Evaluating our work environment.AppliedNursRes. 2016Nov;32:128-33https://doi.org/10.1016/j.apnr.2016.06.006

14. Harvey C, Thompson S, Pearson M, Willis E, Toffoli L. Missed nursing care as an "art form": The contradictions of nurses as carers. Nurs Inq. 2017; 24(3). https:// doi.org/10.1111/nin.12180

15. Schubert M,Clarke SP,Glass TR,Schaffert-Witvliet B,De Geest S.Identifying the thresholds for relationships between impacts of rationing of nursing care and nurse and patient reported outcomes in
Swiss hospitals:a correlational study.Int J Nurs Stud.2009;46(7): 884893. https:// doi.org/10.1016/j.ijnurstu.2008.10.008

16. Schubert M,Clarke SP,Aiken LH,de Geest S.Association between rationing of nursing care and inpatient mortality to Swiss hospitals.Int ] Qual Health Care. 2012; 24(3):230-238. https://doi.org/10.1093/ intqhc/mzs009

17. Hendry C,Walker A.Priority setting in clinical nursing practice:literature review.J Adv Nurs.2004;47(4):427-436

18. Lake S,M oss C,Duke J.Nursing prioritization of the patient need for care:a tacit knowledge embedded in the clinical decision making literature.Int] Nurs Prac 2009;15:376-388

19. Arvidsson E, Andre M, Borgquist L, Carlsson P. Priority setting in Primary health care-dilemmas and opportunities' focus group study.BMC Family Pract.2010;11:71. http://dx.doi.org.manchester. idm.oclc.org/10.1186/1471-2296-11-71

20. Halvorsen K,Forde R,Nortvedt P.The principle of justice in patient priorities in the intensive care unit:the role of significant others.J M ed Ethics. 2009;35(8):483-587. http://dx.doi.org.manchester. idm.oclc.org/10.1136/jme.2008.028183

21. Nortvedt P,Hem M H,Skirbekk H.The ethics of care: role obligations and moderate partiality in health care.Nurs Ethics.2011;18(2): 192-200

22. Evridiki Papastavrou, Panayiota Andreou, Georgios Efstathiou. Rationing of nursing care and nurse-patient outcomes systematic review of quantitative studies. Int J of health planning \& management.2014;29:3-25. https://doi.org/10.1002/hpm.2160

23. VanFossonCA, JonesTL,YoderLH. Unfinished nursing care:an important measure for nursingcaresystems.NursOutlook.2016;64:124-36. https://doi.org/10.1016/j.outlook.2015.12.010

24. Shamseer L, M oher D, Clarke M, Ghersi D, Liberati A, Petticrew M, et al. Preferred reporting items for systematic review and meta-analysis protocols (PRISM A-P) 2015: elaboration and explanation. BMJ. 2015 02; 350:g7647. doi: 10.1136/bmj.g764

25. The Joanna Briggs Institute. Joanna Briggs Institute Reviewer's M anual.2014.The University of Adelaide. 